\title{
A Study of Intense Geomagnetic Storms and their Associated Solar and Interplanetary Causes
}

\author{
S. C. Kaushik \\ Government Autonomous P.G. College, Datiya, M.P., India
}

\begin{abstract}
Shocks driven by energetic coronal mass ejections and other interplanetary transients are mainly responsible for large disturbances in geomagnetic field of Earth and play a key role in producing a geomagnetic storm or substorm. A geomagnetic storm is a global disturbance in Earth's magnetic field usually occurred due to abnormal conditions in the IMF and solar wind plasma emissions caused by various solar phenomenon. Identifying intense geomagnetic storms with Dst decrease more than/or equal to $300 \mathrm{nT}$ occurred during 1981-2001, a correlative study has been performed to analyze the associated solar and interplanetary causes of these 09 events using solar wind plasma, IMF and solar geophysical data. It is observed statistically that $55 \%$ storms have occurred during solar maximum and $45 \%$ occurred during minimum phase of solar cycles. Further, study reveals that $77 \%$ intense storms are associated with CMEs, which confirms earlier findings.
\end{abstract}

Keywords. Sun: coronal mass ejections (CMEs)

\section{Discussion}

JIE ZHANG: You said $77 \%$ of super intense storms are associated with CMEs. I would expect $100 \%$ of them are with CMEs, e.g, for the period with SOHO/LASCO observation. My question is how you get this $77 \%$ number?

KAushik: Yes,this will happen if all the events will be combined together and then study will performed. But because I want to study them separately from 1981 to 2002, the storms associated with CMEs are lesser in numbers. This is also that during solar minima there are coronal holes which are responsible for producing geomagnetic activities, rather than any other cause.

LAKHINA: Comments: I think this division of intense and super intense storm is rather artificial. If both the categories are combined together, the statistics of correlation with CME would go up from $77 \%$ to nearly 100 percent.

KAUSHIK: Yes, that's true by doing so the storms associated with CME will be more in number, but because I wanted to study them separately, i.e., intense and super-intense, that's why I took this division of events, i.e.intense (up to $\sim 250$ to $299 \mathrm{nT}$ ) and super intense ( $\sim 300 \mathrm{nT}$ and above). This is why the CME associated storms' number is low. 